\title{
The utility of self-emulsifying oil formulation to improve the poor solubility of the anti HIV drug CSIC
}

\author{
Nicholas C Obitte ${ }^{1 *}$, Lisa C Rohan², Christianah M Adeyeye ${ }^{3}$, Michael A Parniak ${ }^{4}$ and Charles O Esimone ${ }^{5}$
}

\begin{abstract}
Background: CSIC (5-chloro-3-phenylsulfonylindole-2-carboxamide), a non-nucleoside reverse transcriptase inhibitor (NNRTI) has not been advanced as a therapeutic anti-HIV candidate drug due to its low aqueous solubility and poor bioavailability.

Objective: The objective of this work was to formulate CSIC into self-emulsifying oil formulations for the purpose of improving its aqueous solubility and evaluating in vitro antiretroviral activity.

Methods: CSIC self-emulsifying oil formulations (SEFs) were formulated and evaluated for droplet size, zeta potential, polydispersity index (PDI), viscosity, emulsification time, stability and bioactivity.

Results: Results showed significantly improved solubility of CSIC in the SEFs. The concentration of co-surfactant affected the droplet size, zeta potential and polydispersity index. In vitro bioactivity studies showed that the CSIC SEFs retained full anti-HIV activity.

Conclusion: The in vitro data from this first attempt to formulate CSIC SEFs suggest that improvement on the aqueous solubility of CSIC through this delivery system may accentuate its antiretroviral effectiveness in vivo via bioavailability enhancement. The formulation is therefore intended as an oral anti-HIV agent for prophylactic and therapeutic uses.
\end{abstract}

Keywords: Anti-HIV, Poorly soluble, Self-emulsification, CSIC, Bioactivity, Anhydrous emulsion

\section{Introduction}

Approximately $40 \%$ of all new drug entities have poor aqueous solubility characteristics [1]. Specific to the HIV field a number of newly developed drug candidates including several non nucleoside reverse transcriptase are extremely hydrophobic. Poorly soluble drugs tend to exhibit low bioavailability, high intra- and inter-subject variability, and a lack of dose proportionality, which pose a significant challenge when administered per orally $[2,3]$. Drugs that belong to the Class II Biopharmaceutic classification system are frequently caught up in this web of poor solubility [4]. Enhancement of solubility has been achieved by some drug manufacturers through use of special excipients or formulation carriers that enhance drug dissolution and hence, bioavailability. Several approaches including,

\footnotetext{
* Correspondence: obittenick@yahoo.com

'Department of Pharmaceutical Technology and Industrial Pharmacy, Faculty of Pharmaceutical Sciences, University of Nigeria, Nsukka, Nigeria Full list of author information is available at the end of the article
}

solid dispersions, inclusion complexes, nanoparticles or lipid vehicles [4-6] are available as potential formulation options for hydrophobic drug candidates. An additional option is self-emulsifying oil formulation.

Self-emulsifying oil formulation (SEF) is a lipid composition with inherent capability of improving drug solubility and bioavailability. SEF is a thermodynamically stable isotropic mixture of oil, surfactant and co-surfactant, which on gentle agitation in aqueous medium self-emulsifies to yield micro or nano-emulsion $[7,8]$. SEF, in its native form comes as anhydrous emulsion (emulsion pre-concentrate), but upon dispersion in aqueous medium it transforms into oil-in-water $(\mathrm{O} / \mathrm{W})$ or water-in-oil $(\mathrm{W} / \mathrm{O})$ micro-emulsion or nano-emulsion which constitutes micro-domains of oil droplets stabilized by the interfacial film created by the surfactant and co-surfactant mixture $[9,10]$.

The advantages of SEF over ordinary oil or surfactant dispersions of drugs include the combination of permeability and/or absorption enhancement, thermodynamic stability

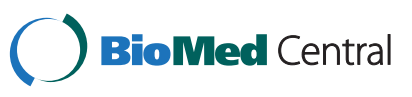


(long shelf life), ease of preparation (zero interfacial tension and formation spontaneity), optical isotropy, ease of sterilization via filtration, high surface area, very small particle size (that promotes adherence to membranes) [11] and the lipoid characteristic which promotes drug intactness in the droplet prior to absorption. Long shelf life, ease of scale-up and manufacturing, improvement on dissolution and lymphatic transport of poorly soluble or lipophilic drugs rate them better than other novel colloidal drug delivery systems [7]. In the SEF front there are commercially available SEF-based antiretroviral drugs which, demonstrate the contribution of SEFs in bioavailability enhancement. Norvir ${ }^{\circledR}$ (ritonavir), Fortavase ${ }^{\circledR}$ (saquinavir) and Aptivus ${ }^{\circledR}$ (tipranavir) are antiretroviral drugs in the market which have been formulated based on this approach $[12,13]$. The dose-limiting side effects associated with hydrophobic antiretroviral drugs may be mitigated if formulated in a SEF. This is because erratic drug release common with poorly soluble drugs $[2,3]$ may sometimes lead to the utilization of excessively high drug dose in traditional formulations. SEF ranks well as a reliable carrier that promotes consistent absorption and bioavailability of hydrophobic drugs $[2,14]$.

NNRTIs such as efavirenz, UC781, dapivirine and 5chloro-3-phenylsulfonylindole-2-carboxamide (CSIC) are potent tight-binding inhibitors of HIV RT, a property that may enhance their utility in both therapeutic and prophylactic use [15]. Efavirenz is poorly soluble, with a bioavailability of $40-45 \%$ and belongs to class II Biopharmaceutic classification system (BCS) [16-18]. Similarly CSIC is poorly soluble and has a limited bioavailability which precludes its further development despite superior in vitro cell-protective ability $[15,19]$.

CSIC pretreatment of uninfected cells to protect them from subsequent HIV infection in the absence of exogenous drug, resulted to sequestration of CSIC in the cell plasma membrane [15]. This may be suggestive of possible high in vivo membrane permeability [20-22]. Nonetheless, the poor aqueous solubility of CSIC may restrict access to absorption through GIT membrane. In this work SEF was explored as a formulation strategy to overcome the limited aqueous solubility and associated bioavailability limitations of CSIC. This is because it offers a nano-sized non-ionic molecular form of the soluble drug which enhances permeability and absorption. Therefore, the objective of this work was to formulate CSIC into SEFs for the purpose of improving its aqueous solubility and evaluating in vitro cell-based antiretroviral bioactivity.

\section{Materials and methods Materials}

The excipients used included triacetin (Acros Organics of Fisher Scientific, U.S.A), lauroglycol $90^{\circledR}$ (propylene glycol monolaurate, Gattefosse, France), and Labrasol ${ }^{\circledR}$ (caprylocaproyl macrogol-8-glycerides, Gattefosse, France). CSIC was obtained from Dr. Michael Parniak at the University of Pittsburgh.

\section{Pseudo ternary phase diagram construction}

Labrasol $^{\circledR}$ (surfactant) and lauroglycol $90^{\circledR}$ (co-surfactant) ratios were varied at five fixed triacetin (oil) ratios $(0.5,1.0$, $1.5,2.0$ and 2.5 ) to generate 30 different oil/surfactant/ co-surfactant homogenous isotropic preconcentrate formulations. Oil (triacetin) concentration was evaluated at levels of $10,20,30,40$, and $50 \%$. The remainder of the formulations studied consisted of a combination of surfactant and co-surfactant. Labrasol ${ }^{\circledR}$ (surfactant) and lauroglycol $90^{\circledR}$ (co-surfactant) were evaluated at levels of 10 to $80 \%$. The titration method was adopted for the determination of self-emulsifying region. A $0.1 \mathrm{~mL}$ quantity of the SEF was pipetted into a $10 \mathrm{~mL}$ beaker. Drop-wise quantities of Milli-Q water (Millipore, USA) filtered through a 0.22 micrometer filter were introduced into the beaker until a stable transparent system was formed. SEF formulations resulting in phase separation or non-transparent emulsions were discarded. The different amounts of the ingredients that contributed to transparent SEFs were determined and a phase diagram plotted using JMP version 4.0.4 software (SAS Institute Inc).

\section{Assay of CSIC}

A liquid chromatography method was used to assay CSIC. A Waters Acquity UPLC was employed. A $1 \mathrm{mg}$ quantity of CSIC was dissolved in $5 \mathrm{~mL}$ of Acetonitrile. From the stock solution serial dilutions were made to attain the concentration range of $10,20,50,100$ and $150 \mu \mathrm{g} / \mathrm{mL}$ respectively. The corresponding Area under the curve values and the resultant calibration curve were determined using the UPLC machine (Acquity UPLC,Waters USA). CSIC was separated with a C18 $(2.1 \times 50 \mathrm{~mm}, 1.7$ micrometer $)$ column using a mobile phase of $0.05 \mathrm{v} / \mathrm{v} \%$ trifluoroacetic acid (TFA) in acetonitrile and $0.08 \mathrm{v} / \mathrm{v} \%$ of TFA in water at a flow rate of $0.4 \mathrm{ml} / \mathrm{min}$. UV detection was at $302 \mathrm{~nm}$. CSIC eluted at $6 \mathrm{~min}$ under these conditions.

\section{Solubility of CSIC in triacetin}

An excess quantity of CSIC was introduced into a vial containing triacetin (oil). The suspension was first vortexed for $10 \mathrm{~min}$ and subsequently mixed intermittently for $5 \mathrm{~h}$ using a mechanical shaker, prior to storage for $24 \mathrm{~h}$. Using a 0.1 micrometer filter (Fisher Scientific, USA) $20 \mu \mathrm{L}$ of the suspension was double-filtered, diluted to $1 \mathrm{~mL}$ with acetonitrile and assayed for CSIC content using the UPLC method described above. A total of seven replicate samples were analyzed. 


\section{Differential scanning calorimetry}

A 2-10 mg quantity of CSIC powder was sealed in a small aluminum pan using a mechanical punch (Perkin Elmer, USA). A sealed empty pan served as control over the temperature range $30-400^{\circ} \mathrm{C}$. The samples were analyzed using the software, Pyris Series- DSC 7. DSC enabled the determination of melting point of CSIC.

\section{Formulation of CSIC SEFs}

From the experiment described under pseudo ternary phase diagram section the optimal SEF formulations were identified as those which did not result in phase separation and maintained transparency. These formulations are detailed in Table 1. CSIC was combined with each

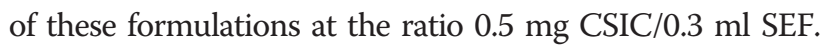
Briefly, $0.5 \mathrm{mg}$ CSIC was mixed with triacetin, and then the appropriate amounts of surfactant and co-surfactant were added sequentially with stirring until a homogenous mix was achieved.

\section{Characterization of CSIC SEFs}

The content of CSIC in each formulation was determined. In these studies a $0.3 \mathrm{~mL}$ quantity of SEF was sampled from each of the five formulations described in Table 1 and diluted to $1 \mathrm{~mL}$ with acetonitrile. After shaking to ensure complete mixing, the mixtures were assayed for CSIC content using the described UPLC method. Each experiment was carried out in triplicates. Additionally viscosity, droplet size, polydispersity index and zeta potential were also determined for each formulation. Viscosity was measured using $0.5 \mathrm{ml}$ samples with a DV-111 ultra programmable Rheometer (Brookfield Engineering Labs, USA) equipped with a CPE 51 Spindle. Data were calculated with Rheocalc V3.1-2 software. Triplicate determinations were made. A Malvern Zeta Sizer (Malvern Instruments Ltd., UK) was used to determine droplet size, polydispersity index and zeta potential. In these studies transparent emulsions were prepared by gentle agitation of the anhydrous SEF with an appropriate quantity of water. Droplet size was evaluated using cuvette containing $2.5 \mathrm{ml}$ of the nano/microemulsion, and zeta potential was measured

Table 1 The $\%$ concentrations (w/w) of the different components in stable and transparent emulsions

\begin{tabular}{ccccc}
\hline Formulation & $\begin{array}{c}\text { Oil:surfactant: } \\
\text { cosurfactant }\end{array}$ & $\begin{array}{c}\text { Oil } \\
(\% \mathbf{w} / \mathbf{w})\end{array}$ & $\begin{array}{c}\text { Surfactant/ } \\
\text { cosurfactant } \\
\text { mix (\%w/w) }\end{array}$ & $\begin{array}{c}\text { Water } \\
(\% \mathbf{w} / \mathbf{w})\end{array}$ \\
\hline A & $10: 80: 10$ & 1.2 & 8.6 & 90.2 \\
B & $10: 70: 20$ & 1.8 & 12.5 & 85.7 \\
C & $20: 70: 10$ & 2.7 & 8.5 & 88.8 \\
D & $20: 60: 20$ & 2.7 & 8.4 & 88.9 \\
E & $30: 60: 10$ & 5.4 & 9.8 & 84.8 \\
\hline
\end{tabular}

using $1 \mathrm{ml}$ in capillary cuvettes. All measurements were performed in triplicates.

\section{Stability studies for CSIC SEFs}

Stability testing for each of the CSIC formulations was evaluated. In these studies each of the CSIC formulations was introduced into vials and stored under three different conditions: (1) $25^{\circ} \mathrm{C}$ and $60 \%$ relative humidity (RH) using a Caron 6010 Humidity Chamber, (2) $40^{\circ} \mathrm{C}$ and $86 \% \mathrm{RH}$ using a Caron 6010 Humidity Chamber, and (3) $50^{\circ} \mathrm{C}$ (Former Scientific Inc., U.S.A). Drug content was determined weekly over a four week period. Additionally stability under stressed conditions was evaluated. Firstly, the effect of centrifugation was studied to evaluate potential metastable conditions, including phase separation and/or drug precipitation. This was done by centrifuging each CSIC formulation at $2000 \mathrm{rpm}$ for $30 \mathrm{~min}$ and visually observing for phase separation and drug precipitation. Secondly, the formulations were also stressed by temperature cycling. In brief, the formulations were subjected to a cycle of $12 \mathrm{~h}$ refrigeration $\left(4^{\circ} \mathrm{C}\right)$ and $12 \mathrm{~h}$ storage at room temperature $\left(25^{\circ} \mathrm{C}\right)$ for a period of one week. Formulations were then evaluated visually for phase separation or drug precipitation.

\section{Emulsification time}

An adaptation of the method of Koo et al. [23] was used. A $0.3 \mathrm{~mL}$ quantity of the SEF was introduced into a beaker containing $200 \mathrm{~mL}$ milli-Q water at $37^{\circ} \mathrm{C}$. The sample was stirred and visually monitored to determine the time for complete emulsification.

\section{Drug release/dispersion studies}

Drug release/dispersion was measured using a Sotax dissolution apparatus (Sotax CP 7, USA). The dissolution chambers were filled with $100 \mathrm{~mL} 0.1 \mathrm{~N} \mathrm{HCl}$. Analyses were carried out at $37^{\circ} \mathrm{C}$ and drug content evaluated at $3 \mathrm{~min}$ intervals over $40 \mathrm{~min}$.

\section{Bioactivity testing}

Two types of bioactivity tests were carried out, (i) standard antiviral assessments in which cells were simultaneously exposed to varying concentrations of drug and HIV, with drug being present throughout the infection process, and (ii) protective or memory effect assessments in which cells were pretreated with varying concentrations of drug for $16 \mathrm{~h}$, then exogenous drug was removed by extensive washing and the cells exposed to HIV in the absence of exogenous drug. HIV replication was evaluated in single replication cycle HIV assays, using P4R5 HIV infection indicator cells (from Dr. John Mellors, University of Pittsburgh). Cells were maintained in DMEM/10\% FBS supplemented with puromycin $(0.5 \mathrm{~g} / \mathrm{mL})$. P4R5 cells express CD4, CXCR4 and CCR5 as well as a $\beta$-galactosidase reporter gene under the control of an HIV LTR promoter [24]. Viral 
infectivity was assessed in 96-well micro-plate assays using P4R5 cells $\left(5 \times 10^{3}\right.$ cells/well). Cells were inoculated with $25 \mathrm{ng}$ HIV-1 p24/well and the extent of infection was evaluated $48 \mathrm{~h}$ post-infection using fluorescence-based $\beta$-galactosidase detection assay. Briefly, infected cells were washed, then incubated with $100 \mathrm{~L}$ lysis buffer (60 mM Na $2 \mathrm{HPO}_{4}, 40 \mathrm{mM} \mathrm{NaH} \mathrm{PO}_{4}(\mathrm{pH} 7.2), 1 \mathrm{mM}$ $\mathrm{MgSO}_{4}, 100 \mathrm{mM}$-mercaptoethanol, 2\% [v/v] Triton X-100) for $1 \mathrm{~h}$ at $37^{\circ} \mathrm{C}$. $\beta$-Galactosidase activity was assessed by addition of $50 \mathrm{~L}$ 4-MUG to a final concentration of $0.5 \mathrm{mM}$, incubation for $1 \mathrm{~h}$ at $37^{\circ} \mathrm{C}$, and then quenched with $150 \mathrm{~L} 0.2 \mathrm{M} \mathrm{Na}_{2} \mathrm{CO}_{3}, \mathrm{pH}$ 11.2. Fluorescence intensity was assessed with a SPECTRA max GEMINI XS dual-scanning micro-plate spectrofluorometer (Molecular Devices, Sunnyvale, CA) using an excitation wavelength of $355 \mathrm{~nm}$ and an emission wavelength of $480 \mathrm{~nm}$, with cutoff filter set to $475 \mathrm{~nm}$.

\section{Statistics}

Results were presented as the mean \pm standard deviation. One-way analysis of variance (ANOVA) was employed in the determination of statistical significance using Graph Pad Instat Demo (Graph pad software, Inc., USA). P $<0.05$ was considered statistically significant.

\section{Results}

\section{Pseudo ternary phase diagram}

Of the 30 formulations tested, only five (A-E) (Table 1) formed transparent micro/nano-emulsions without phase separation. These were evaluated as potential SEFs for CSIC. The pseudo ternary phase diagram is an important preliminary strategy adopted in SEFs to delineate selfemulsifying regions [11]. Preparation of SEFs without a preliminary phase diagram construction may result in loss of formulation stability after a period of storage. The pseudo ternary phase diagram is shown in Figure 1.

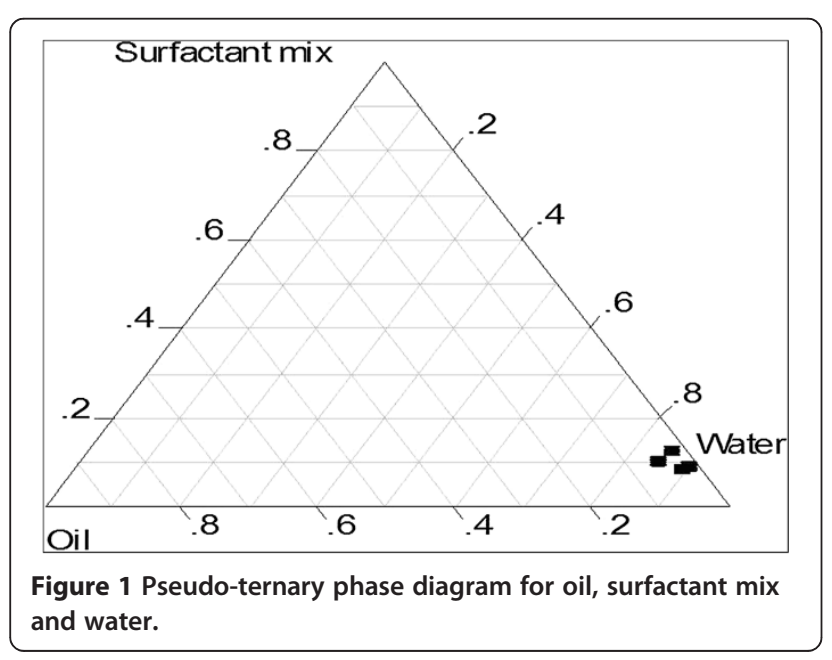

\section{Solubility, stability and drug content}

The mean solubility of CSIC in triacetin was $0.75 \pm$ $0.04 \mathrm{mg} / \mathrm{mL}$. This limited the unit dose of the formulation to $0.5 \mathrm{mg}$ CSIC. Solubility assessments in the surfactants were not done, since the triacetin oil component was the primary determinant for the drug to remain intact in the droplet post-emulsification. The drug content of the SEFs was between $0.42-0.48 \mathrm{mg}$ (83-93\%) (Table 2). Studies to evaluate the effect of temperature and relative humidity on the stability of CSIC in the SEFs showed no substantial changes in CSIC content over the 4 week duration of the experiment (Table 3). Differential scanning calorimetry of CSIC showed a melting peak of $243.67^{\circ} \mathrm{C}$.

Droplet size, polydispersity index, zeta potential, viscosity and emulsification time

One-Way Analysis of Variance (ANOVA)-based TukeyKramer multiple comparisons Test showed that the droplet sizes of placebo and drug-loaded formulations $\mathrm{A}, \mathrm{C}$ and $\mathrm{E}$ respectively were significantly $(\mathrm{p}<0.05)$ smaller than those of formulations B and D (Table 4). Furthermore ANOVAbased Student-Newman-Keuls Multiple Comparisons Test revealed that drug-loading did not significantly $(\mathrm{p}<0.05)$ affect the droplet sizes of the formulations in comparison with placebo batches (Table 4). Placebo formulation D showed significantly $(\mathrm{p}<0.05)$ higher PDI values (Table 4$)$ than those of A, C and E, while B was only significantly $(\mathrm{p}<0.05)$ higher than $\mathrm{C}$ and E. Drug-loaded formulations A, $\mathrm{C}$ and $\mathrm{E}$ recorded significantly $(\mathrm{p}<0.05)$ lower PDI than $\mathrm{B}$ and D. On the contrary, drug-loading did not significantly $(\mathrm{p}<0.05)$ contribute to increased PDI. Low polydispersity indices are preferable as emulsions with higher values may be prone to instability. Negative zeta potential often associated with oil-in-water emulsions was generally the case, with minor variations amongst the batches. The viscosity values of the SEFs were 51mpa.s, 48.8 mpa.s, 44.3 mpa.s, $37.8 \mathrm{mpa} . \mathrm{s}$, and $35.7 \mathrm{mpa}$.s for formulations A through $\mathrm{E}$ respectively. All formulations displayed a Newtonian flow pattern. The rate of emulsification was too fast (within a few seconds) to be accurately measured. The high rate of emulsification precluded determination of a dissolution profile, as the formulations showed $100 \%$ drug release within the earliest time point measured ( 3 minutes).

Table 2 Drug content (\% CSIC recovery) in various SEFs

\begin{tabular}{cccc}
\hline Formulation & $\begin{array}{c}\text { Oil:surfactant: } \\
\text { cosurfactant }\end{array}$ & \multicolumn{2}{c}{ Drug assay content } \\
\cline { 3 - 4 } & & $\mathbf{( m g )}$ & \% recovery \\
\hline A & $10: 80: 10$ & $0.468 \pm 0.0006$ & 93.62 \\
B & $10: 70: 20$ & $0.436 \pm 0.0028$ & 87.12 \\
C & $20: 70: 10$ & $0.453 \pm 0.002$ & 90.58 \\
D & $20: 60: 20$ & $0.449 \pm 0.0002$ & 89.88 \\
E & $30: 60: 10$ & $0.420 \pm 0.001$ & 83.98 \\
\hline
\end{tabular}


Table 3 Thermal stability of CSIC in CSIC SEF

\begin{tabular}{|c|c|c|c|c|c|c|c|c|c|c|}
\hline \multirow{3}{*}{$\mathrm{FC}^{1}$} & \multirow{3}{*}{ Week 0} & \multicolumn{9}{|c|}{ Amount of CSIC remaining $(\mathrm{mg})$} \\
\hline & & \multicolumn{3}{|l|}{ Week 2} & \multicolumn{3}{|l|}{ Week 3} & \multicolumn{3}{|l|}{ Week 4} \\
\hline & & $25^{\circ} \mathrm{C}$ & $40^{\circ} \mathrm{C}$ & $50^{\circ} \mathrm{C}$ & $25^{\circ} \mathrm{C}$ & $40^{\circ} \mathrm{C}$ & $50^{\circ} \mathrm{C}$ & $25^{\circ} \mathrm{C}$ & $40^{\circ} \mathrm{C}$ & $50^{\circ} \mathrm{C}$ \\
\hline A & 0.47 & $0.40 \pm 0.01$ & $0.45 \pm 0.002$ & $0.48 \pm 0.02$ & $0.36 \pm 0.001$ & $0.37 \pm 0.01$ & $0.39 \pm 0.03$ & $0.39 \pm 0.04$ & $0.47 \pm 0.01$ & $0.49 \pm 0.03$ \\
\hline B & 0.44 & $0.40 \pm 0.008$ & $0.45 \pm 0$ & $0.50 \pm 0.1$ & $0.35 \pm 0.01$ & $0.37 \pm 0.02$ & $0.40 \pm 0.01$ & $0.39 \pm 0.02$ & $0.47 \pm 0.02$ & $0.47 \pm 0.02$ \\
\hline C & 0.45 & $0.414 \pm 0.001$ & $0.50 \pm 0.01$ & $0.50 \pm 0.1$ & $0.36 \pm 0$ & $0.39 \pm 0.01$ & $0.38 \pm 0.02$ & $0.40 \pm 0.003$ & $0.48 \pm 0.003$ & $0.46 \pm 0.02$ \\
\hline D & 0.45 & $0.42 \pm 0.004$ & $0.45 \pm 0.01$ & $0.46 \pm 0.01$ & $0.34 \pm 0.02$ & $0.39 \pm 0.02$ & $0.4 \pm 0.01$ & $0.42 \pm 0.004$ & $0.46 \pm 0.01$ & $0.50 \pm 0.001$ \\
\hline E & 0.42 & $0.39 \pm 0.001$ & $0.44 \pm 0.04$ & $0.43 \pm 0.01$ & $0.34 \pm 0.01$ & $0.36 \pm 0.01$ & $0.38 \pm 0.01$ & $0.39 \pm 0.001$ & $0.44 \pm 0.002$ & $0.47 \pm 0.005$ \\
\hline
\end{tabular}

1 = Formulation code.

\section{Bioactivity studies}

In order to establish that the formulation did not result in loss of bioactivity, in vitro anti HIV testing was conducted using the five formulations (A-E). Figure 2 illustrates the antiviral activity for each of the formulations tested. This data showed that the formulations did not result in any loss of antiretroviral bioactivity when compared to unformulated drug substance. Furthermore, the highest concentration tested in these experiments was $0.5 \mu \mathrm{M}$. No toxicity was seen at this level indicating that the SEFs did not result in increased toxicity at the $0.5 \mu \mathrm{M}$ level of dosing. The "memory effect" of the SEFs at $0.1,1.0$, and $10 \mu \mathrm{M}$ was also tested. Cells were incubated with these concentrations for $16 \mathrm{~h}$, then washed and exposed to HIV. All SEFs were very toxic at $10 \mu \mathrm{M}$, and partially toxic at $1 \mu \mathrm{M}$. CSIC alone was not toxic at any of these concentrations. All CSIC samples either tested unformulated or in SEFs gave complete protection without discernible toxicity at $0.1 \mu \mathrm{M}$. The potent protective or memory effect exerted by the SEFs at $0.1 \mu \mathrm{M}$ showed that the cells maintained ready access to the formulated drug, essentially similar to that of the free unformulated CSIC. On the contrary when the placebo SEFs, were tested there was no anti-HIV activity.

\section{Discussion}

In this study it was found that higher oil and lower surfactant ratios (eg, formulation D) provided a narrow transition window from anhydrous to transparent nanoemulsion than did lower oil and higher surfactant ratios (eg, formulation A). Figure 1 clearly portrays a narrow self-emulsifying region, since only a few formulations formed stable micro/nano-emulsions upon titration with water. Out of the 30 batches evaluated only 5 formed transparent emulsions upon aqueous dilution. The rest either witnessed phase separation or formed nontransparent emulsions with outrageous droplet sizes. Thus they were excluded. Our choice of the five stable batches was for comparative purposes.

The determination of the amount of drug that could be dissolved in the anhydrous emulsion is crucial to avoid post-formulation drug precipitation either during storage or following in vivo dispersion in the aqueous GIT environment $[21,25]$. CSIC was found to dissolve very slowly, but once dissolved in the anhydrous emulsion it did not precipitate out. Dissolution in SEF involves molecular interaction with the excipients that renders the crystalline drug amorphous [26]. The $0.75 \mathrm{mg}$ solubility in triacetin motivated the choice of $0.5 \mathrm{mg}$ per dose of SEF. Our bioactivity studies results have confirmed $0.5 \mathrm{mg}$ as pharmacologically effective. However, establishment of minimum effective concentration and unit dose is anticipated in our next investigation.

Experimental evaluations of the thermodynamic stability of SEFs are important to assess the possibility of drug precipitation or phase separation after centrifugation or repeated refrigeration/warming cycles. With conventional emulsions, the index of stability is the absence of phase separation after centrifugation [27], but in SEFs an additional organoleptic index includes the presence/

Table 4 Droplet size, zeta potential and polydispersity index values for the nano/micro-emulsions

\begin{tabular}{|c|c|c|c|c|c|c|}
\hline \multirow[t]{2}{*}{${ }^{1} \mathrm{FC}$} & \multicolumn{3}{|c|}{ Placebo nano/micro-emulsion } & \multicolumn{3}{|c|}{ Drug-loaded nano/micro-emulsion } \\
\hline & $\begin{array}{l}\text { Droplet size } \\
\text { (nm) }\end{array}$ & $\begin{array}{l}\text { Polydispersity } \\
\text { index }\end{array}$ & $\begin{array}{c}\text { Zeta potential } \\
(-)(\mathrm{mV})\end{array}$ & $\begin{array}{l}\text { Droplet size } \\
(\mathrm{nm})\end{array}$ & $\begin{array}{l}\text { Polydispersity } \\
\text { index }\end{array}$ & $\begin{array}{c}\text { Zeta potential } \\
(-)(\mathrm{mV})\end{array}$ \\
\hline A & $40.36 \pm 0.69$ & $0.159 \pm 0.001$ & $0.635 \pm 0.03$ & $67.84 \pm 1.6$ & $0.283 \pm 0.001$ & $0.635 \pm 0.01$ \\
\hline B & $296.5 \pm 54$ & $0.432 \pm 0.16$ & $0.979 \pm 0.002$ & $269 \pm 6.1$ & $0.533 \pm 0.003$ & $1.58 \pm 0.07$ \\
\hline C & $41.1 \pm 0.23$ & $0.134 \pm 0.01$ & $1.32 \pm 0.02$ & $58.6 \pm 0.3$ & $0.283 \pm 0.004$ & $1.72 \pm 0.04$ \\
\hline D & $288.5 \pm 13$ & $0.559 \pm 0.02$ & $1.51 \pm 0.01$ & $286 \pm 11$ & $0.587 \pm 0.001$ & $2.18 \pm 0.08$ \\
\hline E & $52.8 \pm 1.4$ & $0.136 \pm 0.005$ & $0.970 \pm 0.002$ & $65.39 \pm 0.9$ & $0.251 \pm 0.003$ & $2.25 \pm 0.02$ \\
\hline
\end{tabular}

$1=$ Formulation code. 


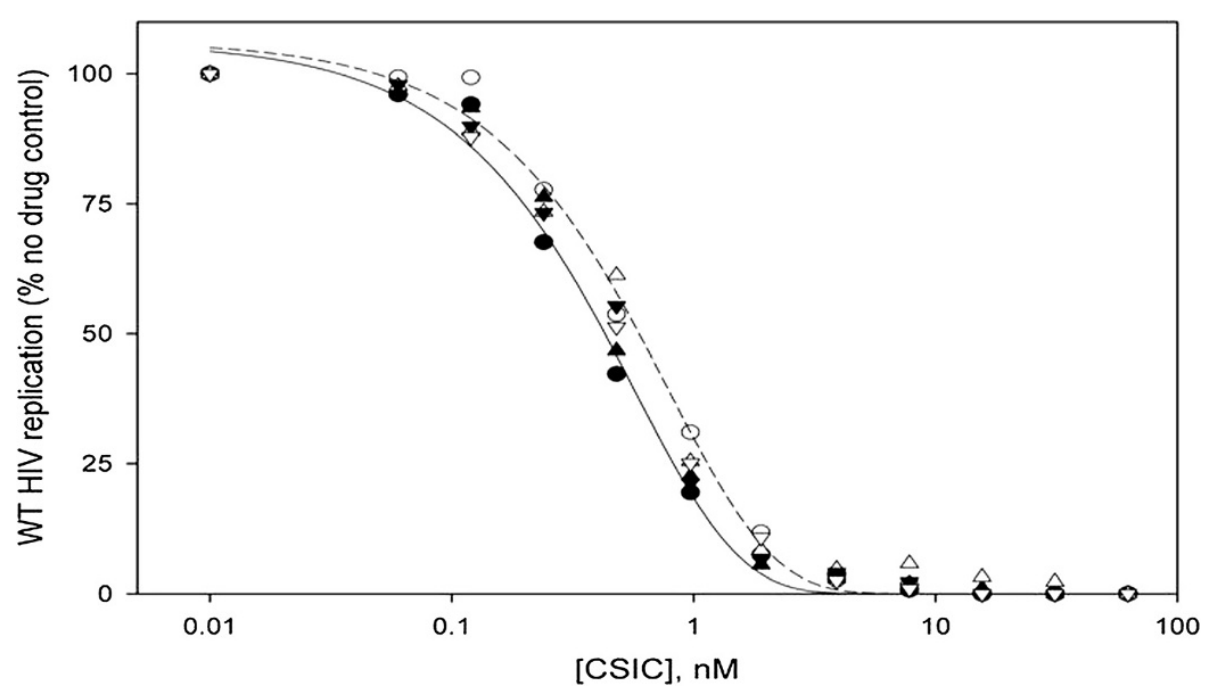

Figure 2 Antiviral activity of CSIC powder and CSIC SEF.

absence of drug precipitate. All the SEFs maintained thermodynamic stability both in the centrifugation and refrigeration/warming cycle protocols. This may be a clue that this formulation strategy is superior to macroemulsions or colloidal formulations [5,11]. Furthermore at different temperature and humidity conditions CSIC demonstrated stability in the SEFs. The high melting point of CSIC (Figure 3) was an indication of thermal stability or non-heat sensitivity. This may be why stability studies lacked evidence of thermal drug degradation.
In all the formulations, as the concentrations of oil and surfactant were simultaneously increased and decreased respectively, co-surfactant was held at a fixed concentration of either $10 \%$ (formulations A, C, E) or $20 \%$ (formulations B, D) (Table 1). It thus appears that droplet size may be related to the co-surfactant concentration used in the formulation; larger droplet sizes resulted from $20 \%$ co-surfactant concentration. The contribution of co-surfactant to droplet size may be connected to its supportive functionality during emulsification, where it

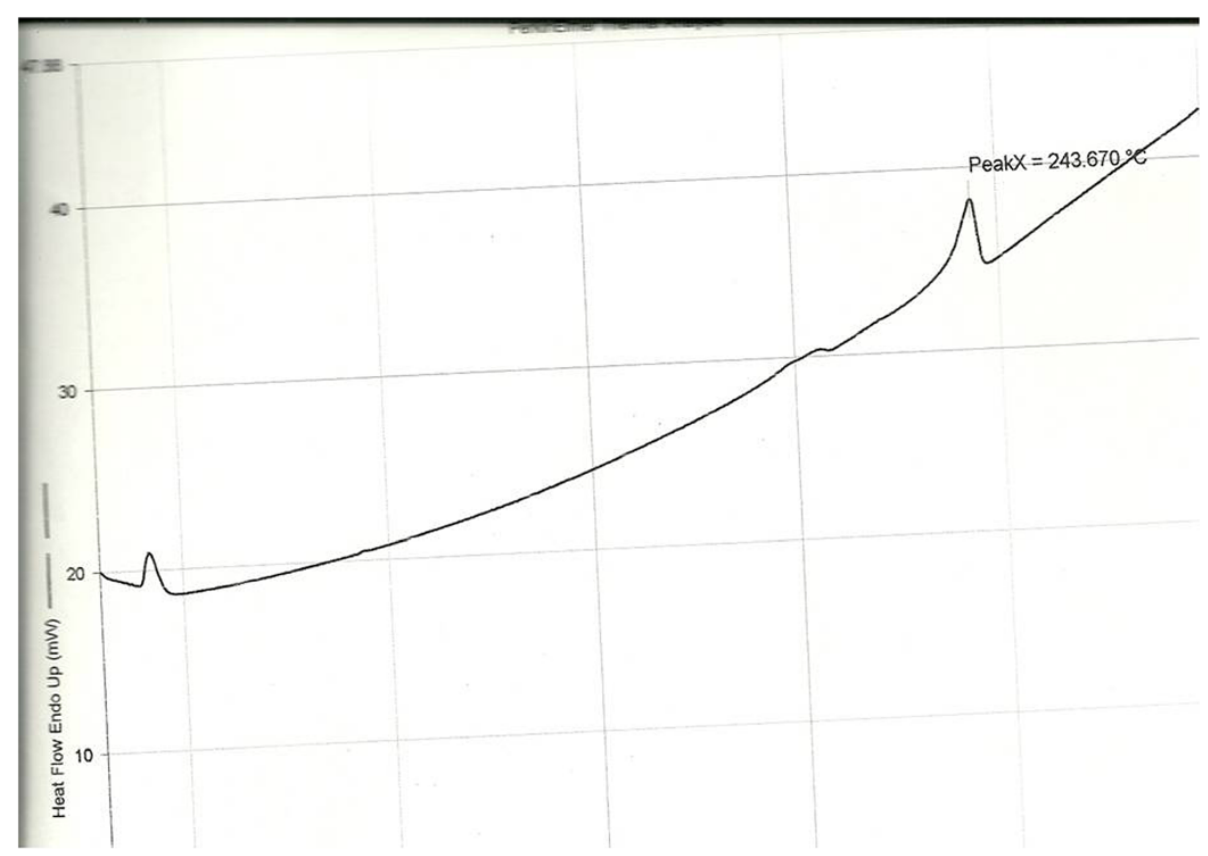

Figure 3 DSC thermogram for CSIC. 
stabilizes the interfacial role of the surfactant. In micro-emulsions, the interfacial tension is so low that the interfacial energy assumes a similar or lower value than the entropy of dispersion. This renders the free energy zero or negative $[28,29]$. The use of only one surfactant may hardly achieve transient negative interfacial tension and fluid interfacial film; hence the need for incorporation of a co-surfactant [30]. Generally, co-surfactants lower the interfacial bending stress and consequently predispose the interfacial film to possess enough flexibility to assume various curvatures required for nano/micro-emulsion formation over a wide range of compositions [11,31]. In addition, lauroglycol $90^{\circledR}$ has a potential dual role of co-surfactant and oil. Higher cosurfactant concentration may have simultaneously increased the interfacial thickness and curvature flexibility of the emulsion droplets with consequent size enlargement. Although the three constituents were miscible, upon emulsification in aqueous phase the cosurfactant with low HLB as the oil may have had better interaction with the oil than the surfactant (with high HLB) and the oil. The polar head of the surfactant may have protruded towards and interacted more with the aqueous medium.

During emulsification more work and energy may be required for droplet formation in high drug-loaded than placebo formulations. Apparently the placebo formulations may ultimately offer lower resistance in aqueous medium during the emulsification process and form smaller droplets than the drug-loaded ones. However in this present investigation the low drug loading $(0.5 \mathrm{mg} /$ dose $)$ did not impel significant droplet size or PDI increase [32] probably because little resistance may have been posed.

The oil constituent of the SEFs may be responsible for the negative zeta potential of the nano/micro-emulsions [33]. The viscosity of SEFs is critical during dispersion in aqueous phase. Higher viscosities tend to slow down the emulsification rate which may be detrimental when faster in vivo drug release and smooth post-absorption bioavailability profiles are crucial. The low values recorded by the SEF formulations in the present study were low enough to preclude the possibility of erratic or slow self-emulsification.

Self-emulsification occurs when the entropy change favoring dispersion is more than the energy needed to increase the surface area of the dispersion $[34,35]$. Two minutes has been suggested as an upper acceptable limit for emulsification time [23]; thus the SEFs of the present work were well within this limit. High emulsification rate was responsible for $100 \%$ drug release within three minutes.

For an orally administered drug, gastrointestinal and pharmaceutical barriers must be overcome before absorption can take place. Aqueous solubility of the drug in question is a crucial factor because only drug in solution is destined for possible absorption [26]. Since the dissolution rate is the rate-determining step to absorption of poorly soluble drugs, improvement on aqueous solubility will promote absorption and bioavailability $[4,36]$. Consequently, only the relative quantity of drug that emerges after systemic absorption may be available at the receptor site for pharmacological activity. The bioactivity investigation conducted within this study attempted to predict the absorption status of CSIC. It did not account for the in vivo gastrointestinal interactions between drug, food and aqueous GIT fluid. The unformulated CSIC had similar antiviral activity as the CSIC SEF formulations. However the CSIC powder was first dissolved in an aqueous solution of dimethyl sulphoxide (DMSO) since it is poorly water soluble. Otherwise, unaided dispersion in water would be unsolubilised and difficult to acces the cells. This may be the predictable in vivo fate when administered orally in that form. The stock solution of the CSIC in DMSO was diluted with excess water and vigorously shaken otherwise drug crystallization would take place. Obviously our SEF formulation offers a less tedious and more commercially viable approach. Two important observations made in our study indicated CSIC SEFs' therapeutic and prophylactic potentials. Bioactivity established at $0.5 \mu \mathrm{M}$ and cell protection at, $0.1 \mu \mathrm{M}$ essentially corroborate drug permeability and activity within the cells. Consequently in the GIT the formulation may witness smooth absorption via the villi of the small intestine. Suffice it to say that of utmost significance was the established improvement on CSIC aqueous solubility which may overcome barrier to its further development as an HIV therapeutic agent $[15,19]$. Poor water solubility of some antiretroviral drugs may predispose to impaired and inconsistent dissolution within the GIT and consequent erratic absorption. This may cause delay in reaching peak plasma concentration and predispose to sublethal drug concentration. Subpharmacological concentrations may induce resistance. The burden of resistance associated with some hydrophobic antiretrovirals may be due to the above reason.

These studies suggest that this formulation approach could address pharmaceutical product development limitations for the anti-HIV drug candidate CSIC such as poor aqueous solubility and cellular permeability. Furthermore this formulation strategy provides a platform which can be extended to other hydrophobic anti-retroviral agents facilitating their advancement in development.

\section{Conclusion}

Further development of CSIC had been stalled due to poor aqueous solubility and low bioavailability. However, in these studies a self-emulsifying oil formulation has been shown to be a reliable formulation approach for 
the improvement of CSIC aqueous solubility. The in vitro antiretroviral bioactivity of the CSIC SEF formulations developed was also established. We therefore conclude that self-emulsifying oil formulation may provide improved bioavailability for this poorly soluble drug making it a viable drug candidate for evaluation in HIV therapy. Further research works are ongoing in our laboratory. Areas of further investigative concern include use of wider spectrum of surfactants, cosurfactants and oils. Extensive stability and bioavailability studies are also contemplated.

\section{Competing interests}

The authors declare that they have no conflict of interest to declare.

\section{Authors' contributions}

ONC, RLC, ACM and ECO were involved in the conception, design and preparation of self-emulsifying oil formulations. All the authors were involved in the preparation and editing of the manuscript. PMA designed and carried out the bioactivity studies. All authors read and approved the final manuscript.

\section{Acknowledgements}

This work was funded in part by the STEP-B project, University of Nigeria, Nsukka, Nigeria and by NIH NIAID grants Al079801 and Al082623. Labrasol ${ }^{\circledR}$ and lauroglycol $90^{\circledR}$ were free samples from Gattefosse, USA. The work was arranged by Dr. Adeyeye to be carried out in Dr. Rohan's lab at the Magee Women's Research Institute at the University of Pittsburgh.

\section{Author details}

'Department of Pharmaceutical Technology and Industrial Pharmacy, Faculty of Pharmaceutical Sciences, University of Nigeria, Nsukka, Nigeria. ${ }^{2}$ Department of Pharmaceutical Sciences, School of Pharmacy, Magee Womens Research Institute, University of Pittsburgh, Pittsburgh, PA, USA. ${ }^{3}$ Department of Biopharmaceutical Sciences, College of Pharmacy, Roosevelt University Schaumburg, Shaumburgh, IL, USA. ${ }^{4}$ Department of Microbiology and Molecular Genetics, School of Medicine, University of Pittsburgh, Pittsburgh, PA, USA. ${ }^{5}$ Department of Pharmaceutical Microbiology and Biotechnology, Faculty of Pharmaceutical Sciences, Nnamdi Azikiwe university, Awka, Anambra, Nigeria.

Received: 25 September 2012 Accepted: 23 May 2013

Published: 31 May 2013

\section{References}

1. Tang B, Cheng G, Gu J, Xu C: Development of solid self-emulsifying drug delivery systems: preparation techniques and dosage forms. Drg Disc Tod 2008, 13:13-14.

2. Kommuru TR, Gurley B, Khan MA, Reddy IK: Self-emulsifyingdrug delivery systems (SEDDS) of coenzyme Q10: formulationdevelopment and bioavailability assessment. Int J Pharm 2001, 212:233-246.

3. Tang J, Sun J, He Z: Self-emulsifying drug delivery systems: Strategy for improving oral delivery of poorly soluble drugs. Curr Drg Ther 2007, 2:85-93.

4. Valizadeh H, Zakeri-Milani P, Barzegar-Jalali M, Mohammadi G, DaneshBahreini M, Adibkia K, Nokhodchi A: Preparation and characterization of solid dispersions of piroxicam with hydrophilic carriers. Drug Dev Ind Pharm 2007, 33(1):45-56.

5. Patel HM, Suhagia BN, Shah SA, Rathod IS, Parmar VK: Preparation and characterization of etoricoxib-b-cyclodextrin complexes prepared by the kneading method. Acta Pharm 2007, 57:351-359.

6. Che E, Zheng X, Sun C, Chang D, Jiang T, Wang S: Drug nanocrystals: a state of the art formulation strategy for preparing the poorly water-soluble drugs. Asian Journ Pharm Scs 2012, 7(2):85-95.

7. Joshi M, Pathak S, Sharma S, Patravale V: Solid microemulsion preconcentrate (NanOsorb) of artemether for effective treatment of malaria. Int J Pharm 2008, 362:172-178.

8. Watnasirichaikul S, Davies NM, Rades T, Tucker IG: Preparation of Biodegradable insulin nanocapsules from biocompatible microemulsions. Pharm Res 2000, 17:6.
9. Lawrence MJ, Rees GD: Microemulsion-based media as novel drug delivery systems. Adv Drug Deliv Rev 2000, 45:89-121.

10. Shafiq S, Faiyaz S, Sushma T, Farhan JA, Khar RK, Ali M: Development and bioavailability assessment of ramipril nanoemulsion formulation. Eur J Pharm Biopharm 2007, 66:227-243.

11. Talegaonkar S, Azeem A, Ahmad FJ, Khar RK, Pathan SA, Khan Zl: Microemulsions: a novel approach to enhanced drug delivery. Recent Pat Drug Deliv Formul 2008, 2:238-257.

12. Pouton $\mathrm{CW}$, Porter JH: Formulation of lipid-based delivery systems for oral administration: materials, methods and strategies. Adv Drug Deliv Rev 2008, 60:625-637.

13. Sha $X, W u$ J, Chen $Y$, Fang $X$ : Self-microemulsifying drug-delivery system for improved oral bioavailability of probucol: preparation and evaluation. Int Journ Nanomed 2012, 7:705-712.

14. Stegemanna S, Leveillerb F: When poor solubility becomes an issue: from early stage to proof of concept. Eur Journ Pharm Sci 2007, 31:249-261.

15. Motakis D, Parniak MA: A tight-binding mode of inhibition is essential for anti-human immunodeficiency virus type 1 virucidal activity of nonnucleoside reverse transcriptase inhibitors. Antim Agent Chem 2002, 6(46):1851-1856.

16. Hari BV, Dhevendaran K, Narayanan N: Development of Efavirenz nanoparticle for enhanced efficiency of anti-retroviral therapy against HIV and AIDS. BMC Infect Dis 2012, 12(Suppl 1):7.

17. Kasim NA, Whitehouse M, Ramachandran C, Bermejo M, Lennernaes $H$, Hussain AS, Junginger HE, Stavchansky SA, Midha KK, Shah VP, et al: Molecular properties of WHO essential drugs and provisional biopharmaceutical classification. Mol Pharm 2004, 1:85-96.

18. Williams TM, Ciccaro TM, MacTough SC, Rooney CS, Balani SC, Condra JH, Emini EA, Goldman ME, Greenlee WJ, Kaufman LR, et al: 5-Chloro-3(phenylsulfonyl)indole-2-carboxamide: a novel, non-nucleoside inhibitor of HIV-1 reverse transcriptase. J Med Chem 1993, 36:1291-1294.

19. Roehr B: Fashioning new tools to deter HIV transmission. J Int Assoc Phys AIDS Care 2000:157-168.

20. Araya $\mathrm{H}$, Tomita M, Hayashi M: The novel formulation design of self-emulsifying drug delivery systems (SEDDS) type o/w microemulsion 11: stable gastrointestinal absorption of a poorly water soluble new compound, ER-1258 in bile-fistula rats. Drug Metab Pharmacokinet 2005, 20(4):257-267.

21. Pouton CW: Formulation of poorly water-soluble drugs for oral administration: physicochemical and physiological issues and the lipid formulation classification system. Europ J Pharm Sci 2006, 29:278-287.

22. Yu LX, Amidon GL, Polli JE, Zhao H, Mehta MU, Conner DP, Shah VP, Lesko $\mathrm{L}$, Chen ML, Lee VH, et al: Biopharmaceutics classification system: the scientific basis for biowaiver extensions. Pharm Res 2002, 19:921-925.

23. Khoo SM, Humberstone AJ, Porter CJ, Edwards GA, Charman WN: Formulation design and bioavailability assessment of lipidicselfemulsifying formulations of halofantrine. Int J Pharm 1998, 167:155-164.

24. Munk C, Brandt SM, Lucero G, Landau NR: A dominant block to HIV-1 replication at reverse transcription in simian cells. Proc Natl Acad Sci USA 2002, 99(21):13843-13848.

25. Patel AR, Vavia PR: Preparation and in vivo evaluation of SMEDDS (Self-microemulsifying drug delivery system) containing fenofibrate. AAPS J 2007, 3:41.

26. Sinha S, Ali M, Baboota S, Ahuja A, Kumar A, Ali J: Solid dispersion as an approach for bioavailability enhancement of poorly water-soluble drug ritonavir. AAPS PharmSciTech 2010, 11(2):518-527.

27. Wilkinson JB, Moore RJ: Emulsions. In Harry's cosmeticology. 7th edn, Burnt Mill Essex UK: George Godwin publishers, Longman House; 1982.

28. Hoar TP, Schulman JH: Transparent water-in-oil dispersions: the oleopathic hydro-micelle. Nature 1943, 152:102-103.

29. Patel MR, Patel RB, Parikh JR, Bhatt KK, Kundawala AJ: Microemulsions: as novel drug delivery vehicle. Latest Reviews 2007, 5:6.

30. Baboota S, Shakeel F, Ahuja A, Ali J, Shafiq S: Design, development and evaluation of novel nanoemulsion formulations for transdermal potential of celecoxib. Acta Pharm 2007, 57:315-332.

31. Kawakami K, Yoshikawa T, Moroto Y, Kanaoka E, Takahashi K, Nishihara Y, Masuda K: Microemulsion formulation for enhanced absorption of poorly soluble drugs, I. Prescription design. J Control Release 2002, 81:65-74.

32. Dixit AR, Rajput SJ, Patel SG: Preparation and bioavailability assessment of SMEDDS containing valsartan. AAPS Pharm Sci Tech 2010, 11(1):314-321. 
33. Gershanik T, Benita S: Positively-charged self-emulsifying oil formulation for improving oral bioavailability of progesterone. Pharm Dev Technol 1996, 1:147-157.

34. Reiss H: Entropy-induced dispersion of bulk liquids. J Colloids Interface Sci 1975, 53:61-70.

35. Lam AC, Schechter RS: The theory of diffusion in microemulsions. J Colloid Interface Sci 1987, 120:56-63.

36. Hecqa J, Deleers M, Fanara D, Vranckx H, Amighi K: Preparation and characterization of nanocrystals for solubility and dissolution rate enhancement of nifedipine. Int J Pharm 2005, 299:167-177.

doi:10.1186/1742-6405-10-14

Cite this article as: Obitte et al.: The utility of self-emulsifying oil formulation to improve the poor solubility of the anti HIV drug CSIC. AIDS Research and Therapy 2013 10:14.

\section{Submit your next manuscript to BioMed Central and take full advantage of:}

- Convenient online submission

- Thorough peer review

- No space constraints or color figure charges

- Immediate publication on acceptance

- Inclusion in PubMed, CAS, Scopus and Google Scholar

- Research which is freely available for redistribution 